\title{
EagleImp: Fast and Accurate Genome-wide Phasing and Imputation in a Single Tool
}

\author{
Lars Wienbrandt ${ }^{1, *}$ and David Ellinghaus ${ }^{1,2}$
}

\author{
${ }^{1}$ Institute of Clinical Molecular Biology, Kiel University, Kiel, Germany and ${ }^{2}$ Novo Nordisk Foundation \\ Center for Protein Research, Disease Systems Biology, Faculty of Health and Medical Sciences, University of \\ Copenhagen, Copenhagen, Denmark \\ *Corresponding author, l.wienbrandt@ikmb.uni-kiel.de (ORCID 0000-0001-5685-2032)
}

\begin{abstract}
Background: Reference-based phasing and genotype imputation algorithms have been developed with sublinear theoretical runtime behaviour, but runtimes are still high in practice when large genome-wide reference datasets are used. Methods: We developed EagleImp, a software with algorithmic and technical improvements and new features for accurate and accelerated phasing and imputation in a single tool. Results: We compared accuracy and runtime of EagleImp with Eagle2, PBWT and prominent imputation servers using whole-genome sequencing data from the 1000 Genomes Project, the Haplotype Reference Consortium and simulated data with more than 1 million reference genomes. EagleImp is 2 to 10 times faster (depending on the single or multiprocessor configuration selected) than Eagle2/PBWT, with the same or better phasing and imputation quality in all tested scenarios. For common variants investigated in typical GWAS studies, EagleImp provides same or higher imputation accuracy than the Sanger Imputation Service, Michigan Imputation Server and the newly developed TOPMed Imputation Server, despite larger (not publicly available) reference panels. It has many new features, including automated chromosome splitting and memory management at runtime to avoid job aborts, fast reading and writing of large files, and various user-configurable algorithm and output options. Conclusions: Due to the technical optimisations, EagleImp can perform fast and accurate reference-based phasing and imputation for future very large reference panels with more than 1 million genomes. EagleImp is freely available for download from https://github.com/ikmb/eagleimp.
\end{abstract}

Key words: Reference-based genotype phasing and imputation; Eagle2; PBWT; GWAS; acceleration; fine- and coarsegrained multi-processing; parallel processing.

\section{Introduction}

Genotype phasing and imputation has become a standard tool in genome-wide association studies (GWAS), and the accuracy of phasing and imputation generally increases with the number of haplotypes from a reference panel of sequenced genomes [1], with the algorithmic complexity (and thus the runtime) of the imputation process depending on the number of unique haplotypes in each genomic segment of the target samples (GWAS input dataset) and the total number of these segments in the reference panel. State-of-the-art reference-based phasing and imputation algorithms such as Eaglez [2], PBWT [3] and minimac4 $[4,5,6]$ have been efficiently developed with runtimes better than linear scaling over the size of the reference panel. For example, Das et al. [5] showed that increasing the reference panel size from 1,092 (1000 Genomes Project Phase 1, 27 million variants) to 33,000 individuals (Haplotype Reference Consortium (HRC), 40 million variants) (>40-fold increase in the number of reference genotypes) only increases the phasing and imputation runtime by a factor of 10 ( $5.3 \mathrm{~h}$ vs. $51.3 \mathrm{~h}$ ) to impute 100 GWAS samples in one single-threaded process. However, this would still result in a runtime of weeks for a new reference panel with more than one million reference samples. The UK Biobank (UKB) has recently made whole-genome sequencing (WGS) data from 200,000 individuals available [7], and the newly established European ' $1+$ Million Genomes' Initiative $(1+M G)[8]$ project is underway, generating WGS data of over one million genomes. The $1+M G$ project will lead to a further increase in the number of reference genotypes by more than $>30$-fold compared to the HRC panel benchmarked by Das et al. [5]. Currently, the best solution to perform phasing and imputation for large datasets is to perform parallel processing on large multi-core systems or high-performance computing (HPC) clusters with hundreds of CPU-cores, to distribute the computational load as much as possible. Therefore, algorithmic and technical improvements together with existing implementations are needed to ensure that phasing and imputation remain feasible for reference panels with more than one million samples.

To allow phasing and imputation for very large reference panels, while ensuring at least the same phasing and imputation accuracy, we developed EagleImp, a software tool for accelerated phasing and imputation. EagleImp introduces algorithmic and implementation improvements to the established tools Eagle2 [2] (phasing) and PBWT [3] (imputation) in a single convenient application. By making changes to the algorithm, parameters, and implementation, we were able to speed up the classical 2-step imputation process with Eaglez and subsequent PBWT by more than a factor of ten for single chromosome analysis and (depending on the parallelisation strategy) at least more than a factor of two for the entire human genome while maintaining or even improving phasing and imputation quality.

EagleImp also provides many new convenient features via simple command line parameters, such as a continuous 
bioRxiv preprint doi: https://doi.org/10.1101/2022.01.11.475810; this version posted January 12, 2022. The copyright holder for this preprint (which was not certified by peer review) is the author/funder. All rights reserved. No reuse allowed without permission.

2 | Wienbrandt, Ellinghaus

progress report to a file, user pre-selection of per genotype imputation information (genotypes, allele dosage, genotype dosage and/or probabilities), phasing confidences and usage information of input variants in a separate file, variant IDs from the reference in imputation output, automated chromosome chunking (if main memory requirements are too high), lock file support (to enable two or more processes to share CPU resources), detection and handling of ref/alt swaps and/or strand flips, the ability to skip certain parts of the algorithm (e.g. pre-phasing, reverse phasing or entire phasing or imputation) and more. In addition, EagleImp supports imputation of chromosome $\mathrm{X}$ and $\mathrm{Y}$ with automatic partitioning by pseudoautosomal regions (PAR).

\section{Background}

Since early studies have shown that the accuracy of imputation increases significantly when the genotype data contains information on the haplotype phase of heterozygous variants [9], it is common practice to apply a haplotype phasing algorithm to a target input dataset prior to genotype imputation. Among others, the best known phasing tools are SHAPEIT2 [10] (phasing without reference) and Eagle2, whereby the latter is currently used on all prominent imputation servers, such as the Sanger Imputation Service (SIS) [11, 1], Michigan Imputation Server (MIS) $[12,13]$ and the newly developed TOPMed Imputation Server (TOPMed) $[14,15]$. Prominent imputation tools are IMPUTE v2 [16], minimac4 [13] and PBWT [3].

In developing EagleImp, we focused on improving and merging Eaglez and PBWT into a single tool. Both tools use the equally named Position-based Burrows-Wheeler Transform (PBWT) data structure introduced by Durbin [3] as their basis. Its main advantages are the compact representation of binary data and the ability to quickly look up any binary sequence at any position in the data. The runtime complexity is linear to the length of the query sequence, independent of the size of the database. To create a PBWT, the algorithm determines permutations of the input sequences for each genomic site such that the subsequences ending at that site are sorted when read backwards. In our work, we propose further algorithmic and implementation improvements that allow a more efficient use of the PBWT data structure and thus increase the speed of phasing and imputation, while maintaining at least the same accuracy of phasing and imputation.

\section{Improvements in EagleImp}

For algorithmic and computational details of the original phasing in Eaglez and imputation in PBWT, we refer to our Supplementary 1 and the original publications by Loh et al. [2] and Durbin [3]. Full details of EagleImp improvements summarised below can be found in the Supplementary 2 .

First, the following points summarise the EagleImp improvements to the data structure and further technical improvements: (i) We have developed a new .qref format for reference data, which significantly improves the reading time of the reference data (Supplementary 2.1). (ii) The PBWT data structure of the condensed reference (Supplementary 1.1) required for each target sample is now stored in a compressed format (Supplementary Figure 1 in Supplementary 2.2), i.e. a binary format (in permuted form) corresponding to the calculated permutation arrays with an index similar to the FM-index used for a Burrows-Wheeler transformation (BWT) [17] (Supplementary Listing 1), to ensure fast generation, compact storage and fast access to the reference data. (iii) Haplotype probabilities are no longer stored in a log-based format and a non-normalised scaling factor is used for the haplotype path probabilities (Supplementary 2.2), which only needs to be up-

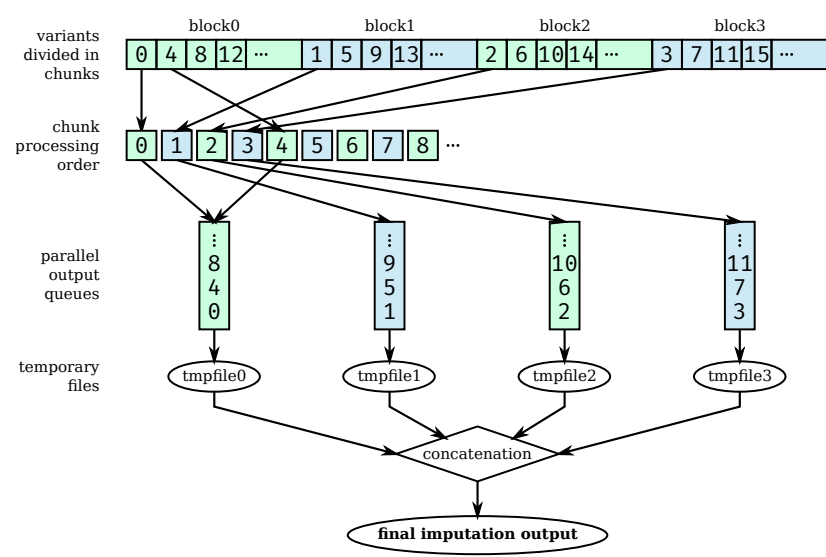

Figure 1. Newly implemented multi-processing scheme for imputation with EagleImp. Variants are distributed over blocks with a separate output file. Chunks in a block are processed repetitively iterating over the blocks. Output files are concatenated at the end. The example shows a distribution over four blocks. The numbers in the chunk indicate the order in which they are processed. See Supplementary 2.3.5 for details.

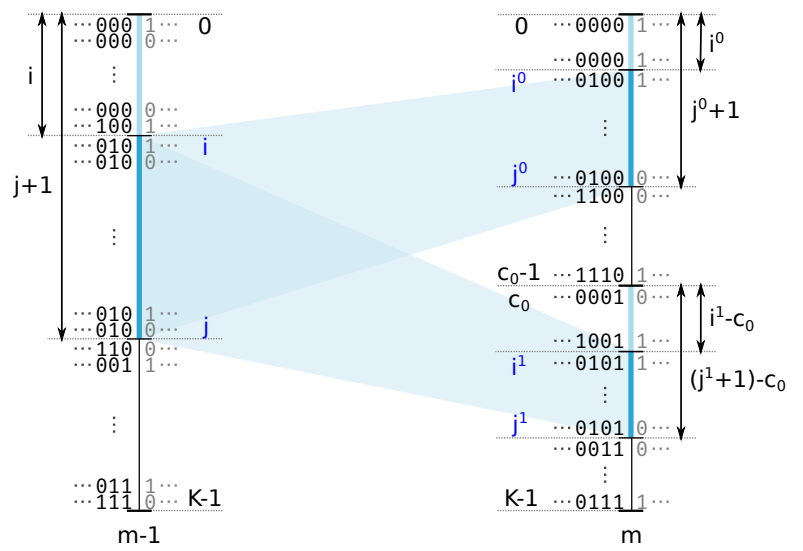

Figure 2. Improved path extension step in EagleImp. Illustration of mapping a PBWT interval $[i, j]_{m-1}$ from position $m-1$ to $m$, resulting in two intervals $\left[i^{0}, j^{0}\right]_{m}$ and $\left[i^{1}, j^{1}\right]_{m}$. The interval $[i, j]_{m-1}$ exemplary represents the sequences in the PBWT that end with 010 at position $m-1$. By stepping forward to position $m$ these sequences are extended either with 0 or 1 and thus are located in one of the mapped intervals $\left[i^{0}, j^{0}\right]_{m}$ or $\left[i^{1}, j^{1}\right]_{m}$ respectively. It is easy to see, that $i=i_{0}+\left(i_{1}-c_{0}\right)$ and $j+1=\left(j_{0}+1\right)+\left(\left(j_{1}+1\right)-c_{0}\right)$ (as $j$ is inclusive in the interval) which directly leads to the mapping equations (6)-(7) in Supplementary 2.1.

dated in case of a predictable loss of precision after several path extension operations. In this way, probability calculations remain precise, especially for heavily needed summations of floating point numbers without an otherwise required approximation (as in Eagle2) or back-transformation. (iv) The imputation of missing genotypes during phasing is obsolete since the subsequent imputation step imputes missing genotypes for shared variants (between target and reference) in the same way as variants that only occur in the reference. To implement this, we used a tree structure to calculate set-maximal matches (Supplementary 2.3). (v) Unlike the original PBWT tool, EagleImp uses multiple threads for genotype imputation, including the use of multiple temporary output files to reduce the input/output (IO) file bottleneck (Figure 1 and Supplementary 2.3). (vi) We introduced a conversion of genotypes and haplotypes into a compact representation with integer registers and made extensive use of Boolean and bit masking operations as well as processor directives for bit operations (such as popcount for counting the set bits in a register) throughout the application which accelerated the computing time significantly (Supplementary Figure 1 and Supplementary Listing 2). 
Second, the following points summarise further algorithmic improvements in EagleImp: (i) Due to the properties of the PBWT data structure, sequences that end with equal subsequences at a certain position are located next to each other in the PBWT and can thus be addressed as an interval. For the path extension step now only the interval boundaries have to be mapped to the next position to get the corresponding intervals for both possible extensions of the sequence (Figure 2 and Supplementary Listing 2). Since the frequency of a subsequence is equal to the (normalised) size of the corresponding interval, the frequency calculation could thus be accelerated. (ii) We increased the default beam width parameter (number of possible paths) from 50 (fixed value in Eagle2) to 128 and the $\Delta$ parameter from 20 (fixed value in Eagle2) to 24 in favor of further increasing the phasing quality with minimal loss of computing time (Supplementary 1.1 and 2.2). (iii) We omitted the identical-by-descent (IBD) check performed by Eaglez before the phasing process, since we found no loss in phasing quality without the IBD-check implemented. (iv) Pre-phasing is disabled by default (unlike Eagle2), as benchmarks showed no improvement (Supplementary 3.4). If desired, pre-phasing can be explicitly enabled with a user option in EagleImp. Likewise, reverse phasing can be optionally disabled.

Third, we introduced new features and improvements in usability, among other things: (i) EagleImp allows imputation of $\mathrm{X}$ and $\mathrm{Y}$ chromosomes by careful handling of haploid samples. Since pseudo-autosomal regions (PAR) are usually encoded with reference to chromosome $\mathrm{X}$ in the target data, we often face the problem of diploid data in the PAR regions and haploid data in the non-PAR region of chromosome $\mathrm{X}$ target (input) files. Existing tools and imputation servers sometimes crash with an error message if diploid and haploid data is mixed in a single input file. We provide a script together with the EagleImp source code that takes care of these regions by automatically splitting the input data with respect to the chromosome X and Y PAR regions before imputation and then merging the imputation results back into one file afterwards. (ii) EagleImp allows reference and alternative alleles in the target to be swapped compared to the reference (with the option --allowRefAltSwap), e.g. an A/C variant is considered as C/A, and it allows strands to be flipped, e.g. an A/C variant is considered a $T / G$ variant at the same chromosomal position (with the option --allowStrandFlip). (iii) EagleImp computes the imputation accuracy $r^{2}$ (as described in Das et al. [18] and used in minimac4) and provides the value together with the allele frequency, the minor allele frequency, the allele count and number as well as the reference panel allele frequency (if available) in the imputation output. An optional $r^{2}$ filter can be applied to filter out variants with low imputation quality. (iv) Phasing confidences and information about how the target variants are used for imputation are provided in separate output files. (v) To save disc space for the output files, the user can decide which information is provided along with the imputed (hard called) genotypes, i.e. any combination of allele dosages (ADS tag), genotype dosages (DS tag), genotype probabilities (GP tag) or no information. Variant IDs in imputation output are provided exactly as they appear in the reference. (vi) EagleImp automatically activates chromosome chunking if the memory requirement is higher than the available main memory (provided as the runtime parameter--maxChunkMem) to eliminate the tedious process of trying out chunk sizes on different input and reference datasets for the user. (vii) For better workload distribution on multi-core computers, we provide a locking mechanism (via a lock file) such that low CPU-load tasks (e.g. reading input files) can run multiple processes at once, while high CPUload tasks (e.g. the phasing and imputation processes) require multiple-exclusion of CPU resources. We provide an optional launch script that uses this feature for simultaneous process-
Table 1. Three different sized reference panels $(A-C)$ were used for phasing and imputation benchmarks.

\begin{tabular}{llccl}
\hline & Reference & \#Variants & \#Samples & Ancestry \\
\hline (A) & HRC1.1 & 40.4 million & 27,165 & mixed \\
(B) & 10ooG Phase 3 & 84.8 million & 2,504 & mixed \\
(C) & synthetic HRC1.1 & 40.4 million & $1,086,600$ & mixed \\
\hline
\end{tabular}

Table 2. 18 target datasets in total were used for phasing and imputation benchmarks to evaluate the runtime and impuation accuracy of EagleImp compared to the original tools Eagle2 and PBWT. Target datasets (1-6) were sampled from the HRC1.1 panel (A) and target datasets (7-16) were sampled from the 10ooG Phase 3 panel (B). For each of the target datasets (1-16), the selected samples were removed from the corresponding reference panels $(A)$ and $(B)$ in the benchmarks to avoid a biased result. The real-world datasets (1718) (from [19]) did not show an overlap of samples with any of the reference panels.

\begin{tabular}{llccl}
\hline & Target & \#Variants & \#Samples & Ancestry \\
\hline$(1)$ & HRC.EUR & 619,872 & 494 & European \\
$(2-6)$ & HRC.v1-5 & 619,872 & 500 & mixed \\
\hline$(7-11)$ & 1kG.EUR.v1-5 & 647,963 & 50 & European \\
$(12-16)$ & 1 kG.v1-5 & 647,963 & 50 & mixed \\
\hline$(17)$ & COVID.Italy & 559,519 & 2,113 & Italian \\
$(18)$ & COVID.Spain & 549,696 & 1,792 & Spanish \\
\hline
\end{tabular}

ing of multiple input files. (viii) A progress indicator shows the progress in percentage (giving the user a hint how long the analysis will take). Optionally, constantly updated status and info files display summarised information about the running process.

\section{Data Description}

To quantify phasing and imputation quality and runtime improvements of EagleImp compared to the original tools Eaglez and PBWT, first, we conducted quality benchmarks with different parameters on three different sized reference panels (Table 1) and 18 target datasets (Table 2) including two realworld target GWAS datasets (from [19]) to further compare imputation accuracy of EagleImp with the accuracy of current imputation servers (SIS, MIS and TOPMed). Then, we ran all runtime benchmarks using the HRC.EUR target dataset (Table 2) and the parameters used for the quality benchmarks. Full descriptions about reference and target datasets from Tables 1 and 2 and details about the setup for quality and runtime comparisons (in particular the preparation of various multiprocessor configurations) can be found in Supplementary 3.

\section{Choice of phasing and imputation parameters}

The exact input parameters for the program call of EagleImp, Eagle2 and PBWT are listed in Supplementary 3.3. For performance comparison, we focused on testing different values of the parameter $K$ (to select the $K$-best haplotypes from the reference for phasing). For example, we ran a benchmark on each HRC.* dataset (Table $2(1-6)$ ) with four different values of $K$ for EagleImp as well as for Eagle2: 10,000 (default setting in Eagle2), 16,384, 32,768, and "max" (where max means using all available haplotypes for phasing, which is 54,330 minus the number of removed haplotypes (988 for target (1) and 1000 for targets (2-6)) in the case of the reduced HRC1.1 panel (A)). All runs used one phasing iteration (reflecting the default setting of Eaglez if the number of target samples is less than half of the number of reference samples). 
bioRxiv preprint doi: https://doi.org/10.1101/2022.01.11.475810; this version posted January 12,2022 . The copyright holder for this preprint (which was not certified by peer review) is the author/funder. All rights reserved. No reuse allowed without permission.
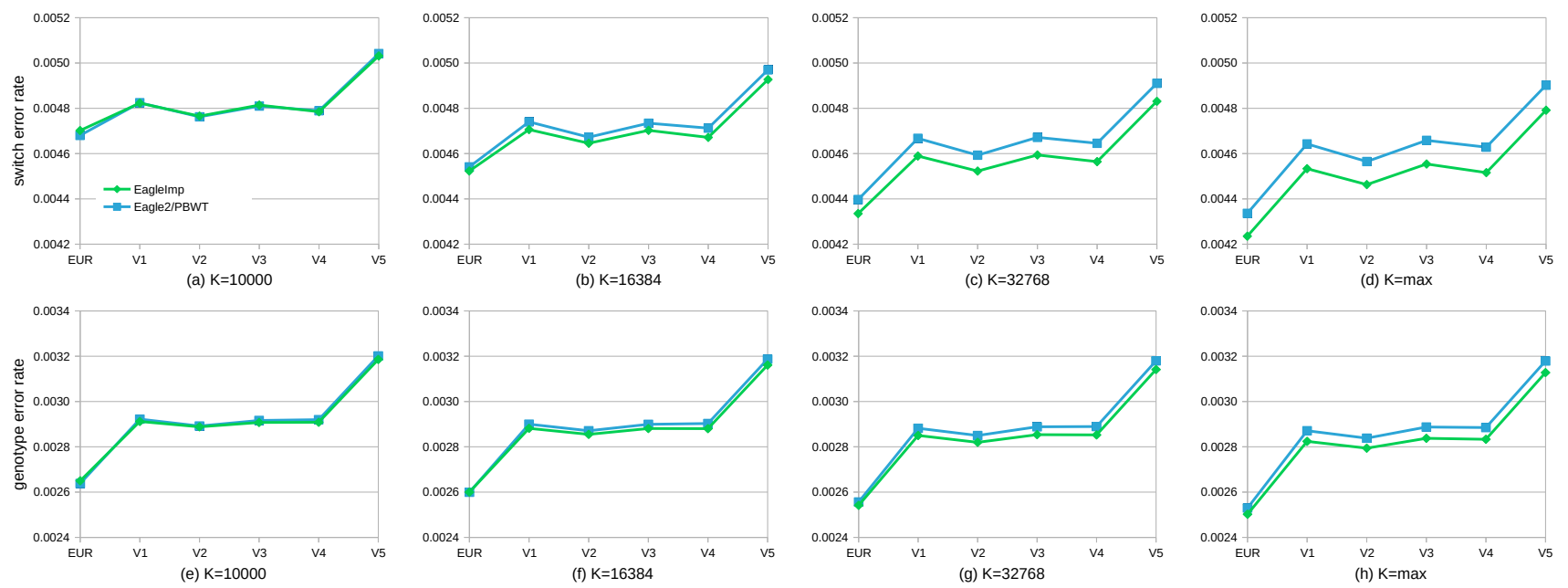

Figure 3. HRC1.1 reference panel (mostly European ancestry; Table 1 (A)) based phasing and imputation performed better for EagleImp (green) compared to the original Eagle2/PBWT (blue) with increasing $K$ in all six test datasets: (a-d) Average (genome-wide) switch error rates (after phasing) and (e-h) genotype error rates (after imputation) using different values of $K \in\{10,000$ (default setting in Eagle2); 16, 384; 32, 768; max $\}$ for six target datasets named HRC.EUR (European ancestry; Table 2 (1)) and HRC.v1-5 (Mixed ancestry; Table 2 (2-6)). The parameter $K$ selects the K-best haplotypes from the reference for phasing, resulting in better quality but longer runtimes.

\section{Benchmark system and program versions}

The computing system we used consists of two Intel Xeon E5$2667 \mathrm{v} 4 \mathrm{CPUs}$, each with 8 cores running at $3.2 \mathrm{GHz}$, resulting in 32 available system threads. The system is equipped with 256 GB of DDR4 RAM and uses a ZFS file system that combines six HDDs, each with a capacity of 2 TB in a raidz2 pool (leading to a total capacity of about $7 \mathrm{~TB})$. The operating system is Ubuntu Linux 21.04 with kernel 5.11.0.

EagleImp is written in $\mathrm{C}++$ and compiled with GCC v10.3.0. For Eaglez and PBWT we used the to date most recent builds on Github: Eagle2 v2.4.1 [20] and PBWT 3.1-v3.1-7-gfog141f [21]. Both tools were also compiled with GCC v10.3.0 together with the required HTSlib v1.12 and bcftools v1.12. For runtime benchmarks, we measured the wall-clock runtime by marking the start and end points of the benchmark with the command date and calculated the difference in runtime.

\section{Metrics for phasing and imputation accuracy}

We counted a phase switch whenever the current phase at a call site differs the phase at the previous call site, and we counted a switch error whenever a phase switch occurs at a calling site in the target but not in the reference when comparing a phased haplotype pair from the target to the original haplotype pair in the reference, or vice versa. The switch error rate per sample is then computed by dividing the switch errors by the number of target variants. In our benchmarks, we showed switch error rate for each benchmark set averaged over all samples.

Genotype errors are determined in a similar way by comparing the genotypes of an imputed haplotype pair with the corresponding genotypes in the reference panel and counting the number of differences. The number of genotype errors in a sample divided by the number of total variants gives the genotype error rate. As with the switch error rate, we calculated the genotype error rate for each benchmark set averaged over all samples.

Another way to determine the imputation quality is the imputation accuracy $r^{2}$, which is a valuable means of interpretation with regard to sample size and statistical power in a GWAS study and which can basically be considered independent of minor allele frequency (MAF) (although the precision of the $r^{2}$ estimate decreases with low MAF) [18]. $r^{2}$ can be estimated for each imputed variant from posterior allele probabilities without knowing the true allele on each chromosome.
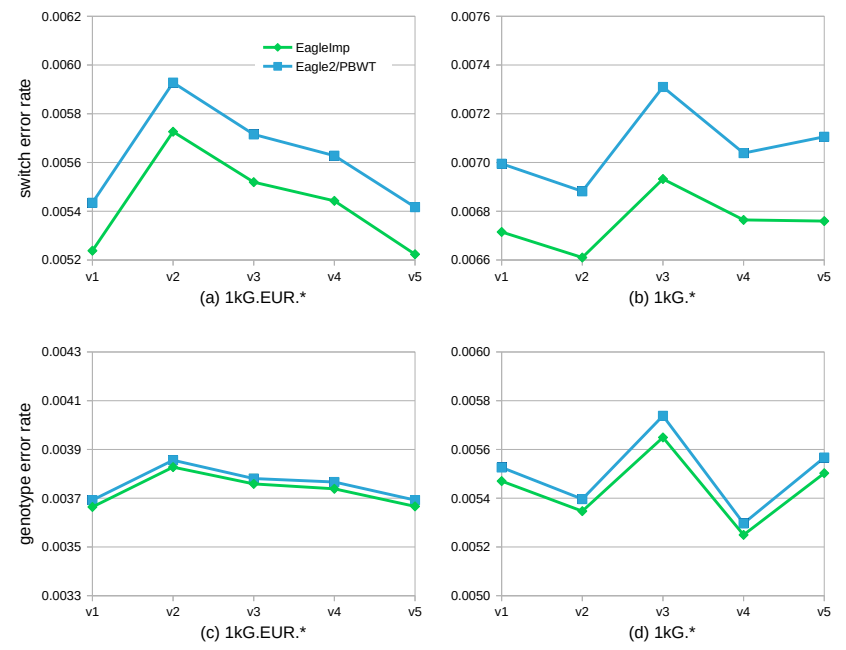

Figure 4. For the 10ooG Phase 3 reference panel (23 global populations; Table 1 (B)), phasing and imputation with EagleImp (green) outperformed the original Eagle2/PBWT (blue) in all ten test datasets: (a-b) Average genome-wide switch error rates (after phasing) and (c-d) genotype error rates (after imputation) for the target datasets named $1 k$ G.EUR.v1-5 (European ancestry; Table 2 (7-11)) and $1 k G . v_{1}-v_{5}$ (different worldwide populations; Table 2 (12-16)). Parameter $K$ was chosen to be maximum in all test runs (here, $K=5,008$ ), thus including the entire $1000 \mathrm{G}$ Phase 3 reference panel.

\section{Phasing and Imputation Quality}

\section{Effect of pre-phasing and reverse phasing on EagleImp}

Contrary to our expectations, pre-phasing in EagleImp resulted in a slight loss of quality for all values of $K$ for the HRC1.1 reference panel (an increased switch error and genotype error rate of about $0.06 \%$ and $0.1 \%$, respectively, (Supplementary Tables 12, Supplementary Figure 2) and at the same time increased the runtime (Supplementary Table 3), we disabled pre-phasing in EagleImp by default. (However, the user can explicitly switch it on again by using the --doPrePhasing switch). In contrast, disabling reverse phasing results in a significant loss of quality for all $K$ (between $3.2 \%$ and $3.7 \%$ increased switch error rate and a genotype error rate increased by approximately $1.0 \%$ to $1.2 \%$ (Supplementary Tables 1-2, Supplementary Figure 2), and should therefore be retained in EagleImp. 

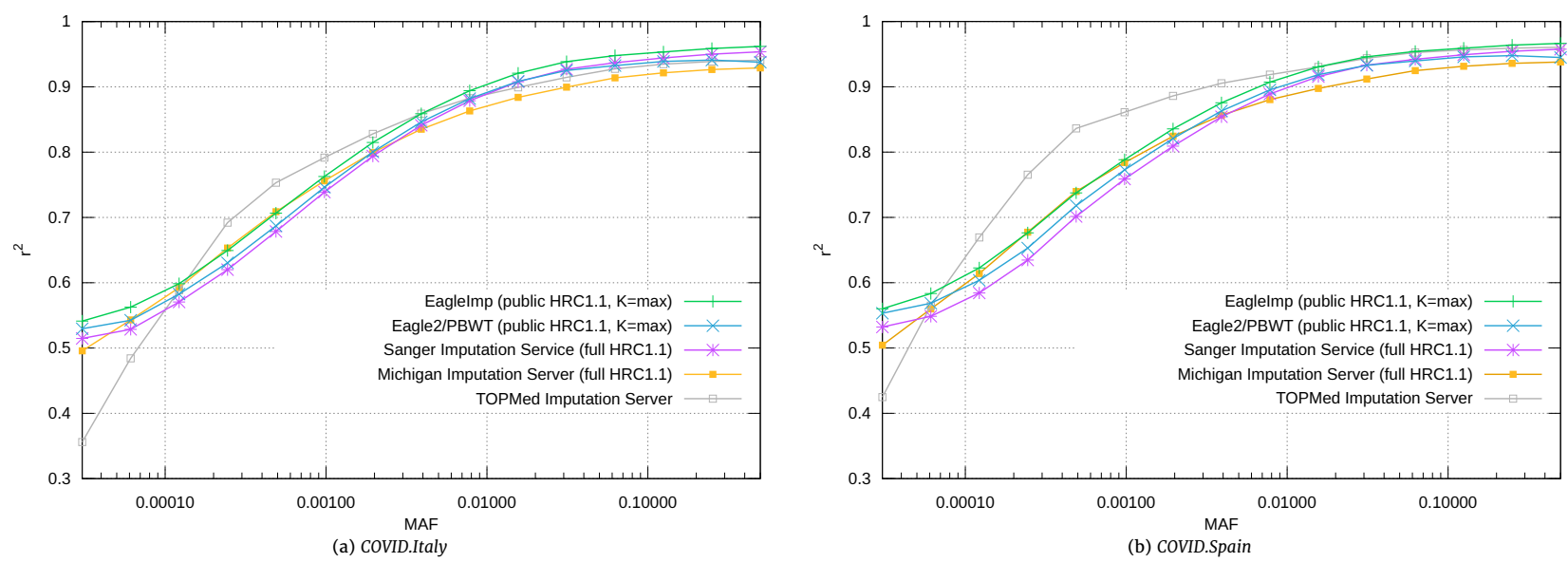

Figure 5. Imputation accuracy $r^{2}$ was higher for EagleImp (green; $K=54,330$ (max)) compared to Eagle2/PBWT (blue; $K=54,330$ (max)) for two real-world GWAS datasets (a) COVID.Italy and (b) COVID.Spain (Table 2 (17-18)), with further comparison to imputation $r^{2}$ from imputation servers SIS (purple), MIS (orange) and TOPMed (grey). EagleImp showed a quality gain compared to TOPMed for minor allele frequency (MAF) greater than 0.004 (COVID. Italy) and 0.01 (COVID.Spain) and achieved at least equivalent results compared to MIS across the entire MAF spectrum, although MIS/SIS and TOPMed use their own reference panels, which are approximately 1.2 times (32,470 samples) and 3.5 times (97,256 samples) larger than the publicly available HRC1.1 panel (27,165 samples; Table 1 (A)) used for EagleImp and Eagle2/PBWT.

\section{Switch and genotype error rates}

For both EagleImp and Eagle2/PBWT, average (genome-wide) switch error rates and genotype error rates decreased (as expected) with higher values of $K$ using the HRC1.1 reference panel (Figure 3; Supplementary Tables 4-5). For $K=10$, 000 the phasing and imputation quality of EagleImp is nearly equal to Eagle2/PBWT (ranging from an increase of $0.5 \%$ and a reduction of $0.2 \%$ in the switch error rate (Figure $3(\mathrm{a})$ ), and an increase of $0.5 \%$ and a reduction of $0.5 \%$ in the genotype error rate Figure $3(\mathbf{e}))$ ). As the value of $K$ increases, the EagleImp runs performed better compared to the corresponding Eagle2/PBWT runs with the same value of $K$ (Figures $3(b-d)$ and Figures $3(\mathbf{f}-\mathbf{h}))$. For example, for $K=16,384$ the switch error rate is lowered between $0.4 \%$ and $0.9 \%$, while for $K=\max$, the switch error rate is lowered between $2.2 \%$ and $2.4 \%$. For the genotype error rate we measure a reduction from $0 \%$ to $0.8 \%$ for $K=16,384$ and from $1.1 \%$ to $1.8 \%$ for $K=\max$. In our $1000 G$ Phase 3 reference benchmark analysis (Figure 4, Supplementary Tables 6-9), EagleImp performed better than Eagle2/PBWT for all 10 target datasets from the $1000 \mathrm{G}$ Phase 3 panel.

Our benchmarks with the HRC1.1 and the 10ooG Phase 3 reference panel showed that switch error and genotype error rates were generally lower in the European input target datasets. As expected, due to the smaller reference panel, the 100oG Phase 3 benchmarks reveal higher error rates than in the HRC1.1 benchmarks. The target datasets with mixed populations showed an increased switch error rate of about $25 \%$ while the genotype error rate is increased by around $40 \%$ to $50 \%$. When compared to Eagle2/PBWT, EagleImp clearly performed better with a reduction of the switch error rate between $4 \%-5 \%$ and the genotype error rate between $0.5 \%-1 \%$.

\section{Imputation accuracy $r^{2}$}

For the real-world GWAS datasets COVID.Italy and COVID.Spain, we determined $r^{2}$ values stratified by MAF (including all variants above this threshold) using the four different $K$ parameters from above (Supplementary Figures 3 and 4). The COVID.Spain dataset showed a generally slightly better imputation performance than the COVID.Italy dataset in all runs, possibly due to a slightly similar genetic background compared to the HRC1.1 reference panel. Already for $K=10,000$ (Supplementary Figure 3a and 4a), phasing and imputation with EagleImp consistently produced higher $r^{2}$ values than the Eagle2/PBWT combination across the entire MAF spectrum, and also higher $r^{2}$ values than the SIS, despite its larger (not freely available) HRC1.1 reference panel as compared to our HRC1.1 benchmark panel. With $K=10,000$ and a MAF greater than 0.001 (and especially at higher MAF), EagleImp also showed a quality advantage in contrast to the MIS, which we attribute to our algorithmic changes. Below a MAF of 0.001, the MIS still seems to show its higher imputation quality due to the larger HRC1.1 reference panel. Especially in this low frequency range, TOPMed imputation (as a reference model) shows that a much larger reference panel plays a another key role in increasing imputation accuracy for rare variants, in addition to algorithmic improvements. Interestingly, for $K=10,000$ and common variants such as in GWAS studies, EagleImp was shown to even achieve a higher quality over TOPMed (here for MAF greater than 0.02 for COVID.Spain and greater than 0.006 for COVID.Italy) probably due to EagleImp's algorithmic improvements, although EagleImp's reference panel is more than three times smaller than that of TOPMed, despite the smaller reference panel in both comparisons.

For higher $K$ parameters, the graphs for EagleImp and Eagle2/PBWT showed (as expected) better $r^{2}$ values but the distance between both tools remained the same (Supplementary Figures $3(b-d), 4(b-d))$. Figure 5 depicts the average genome-wide $r^{2}$ values for the COVID.Italy and COVID.Spain datasets as a function of different MAF thresholds (on logarithmic scale), with $K=\max$ for Eagle2 and EagleImp (which is effectively $K=54,330$ as we used the public HRC1.1 panel). Here EagleImp achieved at least equivalent or better results compared to the MIS across the entire MAF spectrum and showed same quality or a quality gain compared to TOPMed for MAF greater than 0.01 (COVID.Spain) and 0.004 (COVID.Italy).

\section{Phasing and Imputation Runtime}

For the sake of simplicity, we ran all runtime benchmarks using the HRC.EUR target dataset and the parameters used for the HRC1.1 reference benchmarks above, but with different multiprocessing configurations with up to 32 concurrent system threads for EagleImp and Eagle2/PBWT. Details of the various multiprocessor configurations tested can be found in Supplementary 3.7. In addition, we examined runtimes of individuals chromosomes processed with all 32 system threads (referred to as $1 \times 32$ runs) (Supplementary 3.8) and single-thread 
bioRxiv preprint doi: https://doi.org/10.1101/2022.01.11.475810; this version posted January 12, 2022. The copyright holder for this preprint (which was not certified by peer review) is the author/funder. All rights reserved. No reuse allowed without permission.

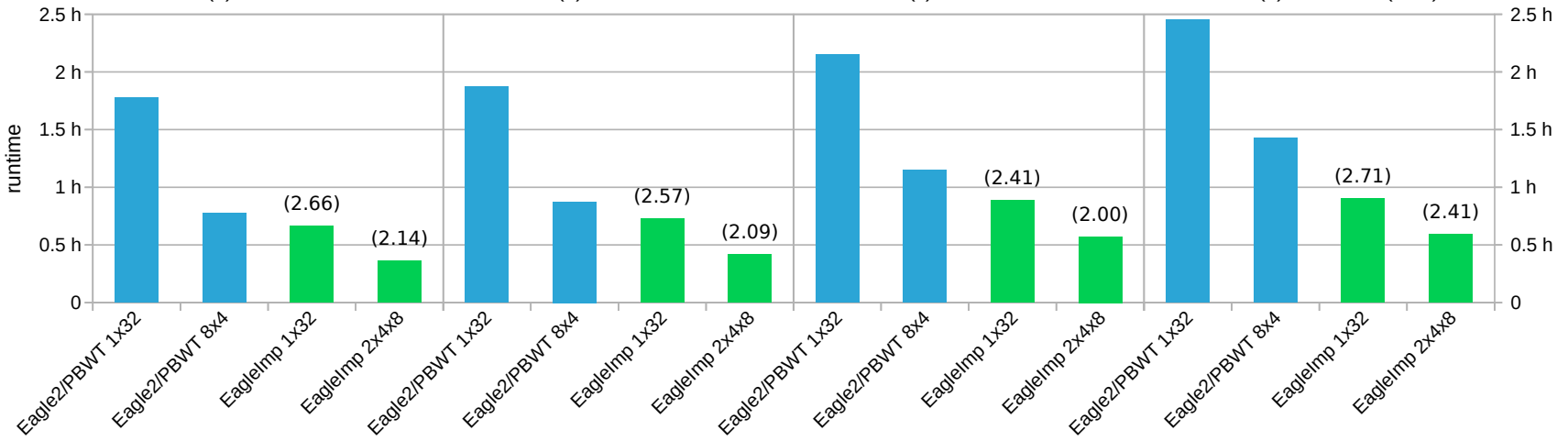

Figure 6. Faster runtimes of EagleImp compared to Eagle2/PBWT (with at least the same accuracy, Figure 3), demonstrated for the processing of 494 GWAS target samples using the HRC1.1 reference panel (Table 1(A)) and different $K$ parameters ( $K=54,330$ denotes the maximum value including all haplotypes from the HRC1.1 panel). The naive multi-processor configuration (1X32 with 32 phasing threads and each chromosome processed sequentially) and the best individual multiprocessor configuration (i.e. fastest individual configuration determined from 6 different runs of Eagle2/PBWT and 8 different runs of EagleImp, see Supplementary Table 10) were compared. The numbers in brackets give the acceleration factor of EagleImp compared to the corresponding Eagle2/PBWT run.

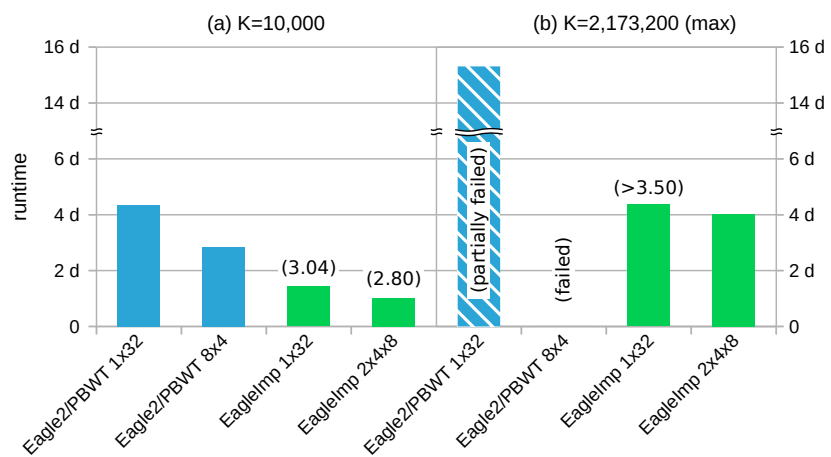

Figure 7. Phasing and imputation runtimes for the synthetic HRC1.1 reference panel (Table 1 (C)) containing >1 million samples (2,173,200 haplotypes) and the real-world COVID.Italy dataset (2,113 samples; Table 2 (17)). The numbers in brackets give the acceleration factor of EagleImp compared to the corresponding Eagle2/PBWT run. For $K=$ max Eaglez could not complete the analysis in $8 \times 4$ configuration due to insufficient main memory (marked with "failed"), hence, no runtime or speedup could be measured in this configuration. For the 1X32 configuration phasing with Eagle2 crashed for chromosomes 1-4 and 6 ("partially failed") and the stated runtime includes the incomplete runs.

runtimes with multi-processing disabled (Supplementary 3.9), since multi-processing strategies can behave differently on different computing systems. We also measured the runtimes of our real-world GWAS datasets COVID.Italy and COVID.Spain which can be found in Supplementary 3.10. Note, that the runtime benchmarks do not include the preparation of the reference files (Supplementary 3.11), which is required for PBWT and is optional for EagleImp. (PBWT requires a .pbwt file for each reference file; for EagleImp we used our newly developed .qref format instead of .vcf.gz or .bcf.)

\section{Multi-processing runtimes}

We observed that for any value of $K$, the Eagle2.8x4 configuration performed best for the Eagle2/PBWT runs and

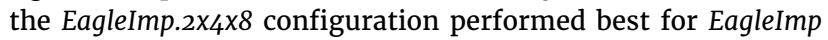
(Supplementary Table 10), which is why we only compared these two configurations in the following.

For $K=10$, 000 (Figure 6 (a)), Eagle2.1×32 took 1 hour and 46 minutes: The Eagle2.8x4 configuration accelerated this by a factor of 2.30 to 46 minutes. In contrast, the EagleImp.1 $1 \times 32$ configuration required 40 minutes, which we could speed up

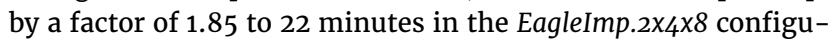
ration. This corresponds to a speedup factor of 2.66 when comparing the $1 \times 32$ configurations of Eagle2/PBWT and EagleImp or a factor of 2.14 when comparing the fastest multi-processing configurations of the two tools. The total runtime increased with higher values of $K$ (Figure $6(b-d)$ ), but the speedup factors between both tools only varied slightly. For $K=\max$, the Eagle2/PBWT runtime was 2 hours and 27 minutes for the $1 \times 32$ configuration and 1 hour and 25 minutes for $8 \times 4$. EagleImp analysed the same data in 54 minutes ( $1 \times 32)$ and 35 minutes ( $2 \times 4 \times 8)$, resulting in speedup factors of 2.71 and 2.41 , respectively.

When comparing runtimes for individual chromosomes, we additionally measured runtimes for phasing-only, imputationonly and combined runs. For the phasing-only runs, we observed that EagleImp is between 2.93 and 4.81 times faster than Eagle2 for all chromosomes and different values of $K$ (Supplementary Table 11). The imputation-only runs of EagleImp with 32 threads when compared to PBWT showed the advantages of the multi-threading capability of EagleImp (which PBWT does not offer), with a speedup between 7.77 and 12.94 (Supplementary Table 12). The combination of phasing and imputation offers the advantage for EagleImp that the reference panel does not have to be read twice (as is the case with Eagle 2 and $P B W T$ ), resulting in a combined speedup between 5.82 to 10.81 for single chromosomes (Supplementary Table 13).

\section{Single-thread runtimes}

We measured the runtimes of the HRC.EUR dataset from above with the four values of $K$ exemplary for chromosome 2 and chromosome 21 (largest and smallest number of input variants) with only one thread for Eagle2 and EagleImp (by using the corresponding --numThreads parameters for both tools). We found that EagleImp is faster than Eagle2/PBWT with measured speedups between 1.59 and 2.48 for phasing only and between 1.40 and 1.51 for imputation only, and a combined speedup between 1.58 and 2.25 (Supplementary Tables 14-15).

\section{Phasing and Imputation using One Million Reference Genomes}

To our knowledge, the TOPMed imputation server uses the largest reference panel currently available for phasing and imputation, with 97,256 reference samples (TOPMed $r$ ) [15]. To explore EagleImp's capabilities in handling a future reference panel with more than one million samples, we created a synthesised panel synthetic HRC1.1 (1,086,600 samples and 40.4 million variants, Table 1 (C)) by multiplying the sample data of the public HRC1.1 panel (Table 1 (A)) 40 times (see section Data Description). We measured EagleImp's and Eagle2/PBWT's runtimes for the real-world GWAS dataset COVID.Italy $(2,113$ samples) using $K=10,000$ and $K=2,173,200$ (maximum $K$ 
from the synthetic HRC1.1 panel) and the naive (Eagle2.1x32, EagleImp.1X32) and respectively fastest multi-processor configu-

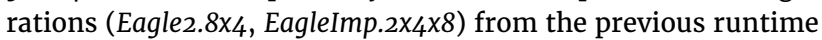
benchmark (Supplementary Table 18).

EagleImp outperformed Eagle2/PBWT with a speedup factor of at least $2.80(K=10,000)$ and $3.50(K=\max )$ (Figure 7). EagleImp took about 24 hours for $K=10,000$ in $2 \times 4 \times 8$ configuration, while, in contrast, the original Eagle2/PBWT took almost three days (68 hours) in $8 \times 4$ configuration respectively. The runs with $K=\max$, i.e. $K=2,173,200$ in this case, resulted in a runtime of 4 days for EagleImp in $2 \times 4 \times 8$ configuration, while Eagle2 could not complete the run due to insufficient memory and missing automatic chunking ability (requirement of more than 256GB of RAM) on our benchmark system in $8 \times 4$ configuration. In the $1 \times 32$ configuration Eagle2 crashed only for the larger chromosomes 1-4 and 6 with a total runtime (including the crashes) of more than 15 days.

\section{Discussion}

We have introduced EagleImp, a fast and accurate tool for phasing and imputation. Due to technical improvements and changes in the data structure, EagleImp is 2 to 10 times faster (depending on the multi-processor configuration) than $\mathrm{Ea}$ gle2/PBWT, with same or better phasing and imputation quality in all tested scenarios. For common variants investigated in typical GWAS studies, EagleImp also yielded equal or higher imputation accuracy than the imputation servers MIS, SIS and TOPMed that use larger (not freely available) reference panels, which we attribute to our algorithmic improvements. Because of the technical optimisation and the improvement of the stability of the software, EagleImp can perform phasing and imputation for upcoming very large reference panels with more than 1 million genomes.

For phasing, we accelerated the search for the $K$ best haplotypes, the generation of the condensed reference and the entire phasing process by using an alternative haplotype encoding, Boolean comparison operations and processor directives for bit operations. We improved the accuracy of the phase probability calculation by using a scaled floating point representation instead of a logarithm-based representation, and we improved the frequency lookups in the PBWT data structure by introducing our interval mapping procedure and omitting duplicate calculations of frequencies in the beam search. We investigated the effects of pre-phasing and reverse phasing procedures with the conclusion that pre-phasing is unnecessary. By introducing multi-threading and writing multiple temporary files during imputation, and by using a tree-structure and the same interval mapping technique as for the phasing part to search for set-maximal matches, we were able to speed up the imputation process considerably compared to PBWT. Furthermore, the correct treatment of missing genotypes from the input dataset became possible with the tree-structure.

An additional reduction in computing time was made possible by the introduction of the Qref-format (producible from standard .vcf or .bcf files) for fast reference loading, by combining phasing and imputation in a single tool, and by an helper script that introduces multi-processing with several worker processes and a balanced distribution of a complete genome-wide input dataset. We further enhanced the usability of phasing and imputation by adding several convenience features, such as chromosome $\mathrm{X}$ and $\mathrm{Y}$ handling, imputation $r^{2}$ and MAF calculation for output files, user selection of desired imputation information (allele dosages, genotype dosages, genotype probabilities), automated optimisation of chromosome partitioning (chunking) and memory management during runtime, preserving variant IDs from reference, $r^{2}$ filtering and other things.

Unfortunately, we were not able to sufficiently investigate the quality of the imputation of rare variants, as we did not have the larger reference panels of the MIS, SIS and the TOPMed imputation servers at our disposal. We noticed that in the imputation of common variants, the MIS (uses minimac4 for imputation) fell behind the SIS (uses PBWT for imputation) in terms of quality, despite the same larger HRC reference panel, but the MIS performed better than the SIS in the imputation of rare variants. We have not investigated this further. However, since new, even larger reference panels will be available soon, this difference should not matter too much. We already designed EagleImp to use more than one million reference genomes ( $>2$ million haplotypes) for reference-based phasing and imputation. In this case, EagleImp will be able to show its full strength in terms of runtime and quality compared to other tools, although again an optimisation of the $K$ parameter (selection of the $K$ best reference genomes where a higher $K$ increases the runtime but produces better results) will be required. Imputation in combination with new methods such as study-specific pre-selection of reference samples using deep learning imputation reconstruction methods for reference panels [22] could provide a higher accuracy for rare variants.

As an improvement, in the future we plan to replace the slow HTSlib with our own library in order to speed up the writing process since this is still a bottleneck in EagleImp. A reduction of the runtime by using Field Programmable Gate Arrays (FPGAs) is another possibility to reduce the runtime even further (Wienbrandt et al. [23]). Furthermore, we plan to offer a free web service for EagleImp, so that the advantages of EagleImp can also be used by the community even without special hardware equipment.

\section{Methods}

We give detailed information about the original concepts of $\mathrm{Ea}$ glez [2] and PBWT [3] in Supplementary 1 and describe our changes to the algorithm and implementation in Supplementary 2, with additional details about the performed benchmarks in Supplementary 3.

\section{Availability of source code and requirements}

\author{
- Project name: EagleImp \\ - Project home page: https://github.com/ikmb/eagleimp \\ - Operating system: Linux \\ - Programming language: $\mathrm{C}++$ (bash, awk) \\ - Other requirements: HTSlib, Zlib, BOOST, TBB, Cmake \\ - License: GNU GPL v3.0
}

\section{Availability of supporting data and materials}

All quality and runtime measures from our benchmarks are listed in the Supplementary Material. The 1000 Genomes Phase 3 reference panel can be downloaded at ftp://ftp.1000genomes. ebi.ac.uk/vol1/ftp/release/20130502/. Data access to the EGAD00001002729 dataset for the HRC1.1 panel is restricted and was granted under request ID 11699. The benchmark datasets HRC.EUR, HRC.v1-5, 1 kG.EUR.v1-5 and $1 k$ G.v1-5 are subsets of the previously mentioned reference panels. 
bioRxiv preprint doi: https://doi.org/10.1101/2022.01.11.475810; this version posted January 12,2022 . The copyright holder for this preprint (which was not certified by peer review) is the author/funder. All rights reserved. No reuse allowed without permission.

8 | Wienbrandt, Ellinghaus

\section{Declarations}

\section{List of abbreviations}

BCF Binary Variant Call Format

BWT Burrows-Wheeler Transform

HRC Human Reference Consortium

PBWT Position-based Burrows-Wheeler Transform

VCF Variant Call Format

\section{Ethical Approval}

All participants provided written informed consent, and the study was approved by the ethics boards of the participating institutions in agreement with the Declaration of Helsinki principles.

\section{Competing Interests}

The authors declare that they have no competing interests.

\section{Funding}

The project received fundings from the DFG (Deutche Forschungsgesellschaft) grant no. EL 831/3-1, PI: David Ellinghaus, and grant no. WI 4908/1-1, PI: Lars Wienbrandt. This work was supported by the German Federal Ministry of Education and Research (BMBF) within the framework of iTREAT (grant 01ZX1902A). The study received infrastructure support from the DFG Cluster of Excellence 2167 "Precision Medicine in Chronic Inflammation (PMI)" (DFG Grant: "EXC2167") and the DFG research unit "miTarget" (Projectnummer 426660215).

\section{Author's Contributions}

Lars Wienbrandt Application software development; hardware infrastructure; benchmark setup, execution, analysis, interpretation; manuscript writing

David Ellinghaus Data acquisition; benchmark analysis, interpretation; manuscript writing; project supervision

\section{Acknowledgements}

The authors would like to thank the COVID-19 GWAS Group for the use of the COVID-19 GWAS data for the benchmark.

\section{References}

1. McCarthy S, Das S, Kretzschmar W, Delaneau O, Wood AR, Teumer A, et al. A reference panel of 64,976 haplotypes for genotype imputation. Nature Genetics 2016 Aug;48(10):1279-1283.

2. Loh PR, Danecek P, Palamara PF, Fuchsberger C, Reshef YA, Finucane HK, et al. Reference-based phasing using the Haplotype Reference Consortium panel. Nature Genetics 2016 Oct;48:1443-1448.

3. Durbin R. Efficient haplotype matching and storage using the positional Burrows-Wheeler transform (PBWT). Bioinformatics 2014;30(9):1266-1272.

4. Fuchsberger C, Abecasis GR, Hinds DA. minimac2: faster genotype imputation. Bioinformatics 2014 Oct;31(5):782784. https://doi.org/10.1093/bioinformatics/btu704.

5. Das S, Forer L, Schönherr S, Sidore C, Locke AE, Kwong A, et al. Next-generation genotype imputation service and methods. Nature Genetics 2016 Aug;48(10):1284-1287.

6. Ketian Yu SD, Minimac4, https://genome.sph.umich.edu/ wiki/Minimac4;.

7. Bycroft C, Freeman C, Petkova D, Band G, Elliott LT, Sharp $\mathrm{K}$, et al. The UK Biobank resource with deep phenotyping and genomic data. Nature 2018 Oct;562(7726):203-209.

8. European Commission, European '1+ Million Genomes' Initiative, https://digital-strategy.ec.europa.eu/en/ policies/1-million-genomes; 2021.

9. Browning SR. Missing data imputation and haplotype phase inference for genome-wide association studies Hum Genet 2008 Dec;124(5):439-50.

10. Delaneau O, Zagury JF, Marchini J. Improved whole chromosome phasing for disease and population genetic studies. Nature Methods 2013;10:5-6.

11. Wellcome Sanger Institute, Sanger Imputation Service, https://imputation. sanger.ac.uk/;.

12. US National Institutes of Health, Michigan Imputation Server, https: //imputationserver.sph.umich.edu/;.

13. Das S, Forer L, Schönherr S, Sidore C, Locke AE, Kwong A, et al. Next-generation genotype imputation service and methods. Nature Genetics 2016 Aug;48:1284-1287.

14. National Heart, Lung, and Blood Institute, BioData Catalyst TOPMed Imputation Server, https://imputation. biodatacatalyst.nhlbi.nih.gov/;

15. Taliun D, Harris DN, Kessler MD, Carlson J, Szpiech ZA, Torres R, et al. Sequencing of 53,831 diverse genomes from the NHLBI TOPMed Program. bioRxiv 2019;https: //www.biorxiv.org/content/early/2019/03/06/563866.

16. Howie BN, Donnelly P, Marchini J. A flexible and accurate genotype imputation method for the next generation of genome-wide association studies. PLoS Genet 2009 Jun;5(6).

17. Ferragina P, Manzini G. Opportunistic Data Structures with Applications. In: Proc. of FOCS2000; 2000. p. 390398.

18. Das S, Abecasis GR, Browning BL. Genotype Imputation from Large Reference Panels. Annu Rev Genomics Hum Genet 2018 Aug;19:73-96.

19. Ellinghaus D, Degenhardt F, Bujanda L, Buti M, Albillos A, Invernizzi $\mathrm{P}$, et al. Genomewide association study of severe COVID-19 with respiratory failure. The New England Journal of Medicine 2020 Oct;383(16):1522-1534.

20. Loh PR, Price AL, EAGLE v2.4.1 (18 Nov 2018), https:// github.com/poruloh/Eagle.git;

21. Durbin R, Danecek P, PBWT 3.1-v3.1-7-gfog141f (15 Nov 2017), https://github.com/VertebrateResequencing/ pbwt.git;.

22. Shi S, Qian Q, Yu S, Wang Q, Wang J, Zeng J, et al. RefRGim: an intelligent reference panel reconstruction method for genotype imputation with convolutional neural networks. Brief Bioinform 2021 Nov;22(6):bbab326.

23. Wienbrandt L, Kässens JC, Ellinghaus D. Reference-Based Haplotype Phasing with FPGAs. In: Krzhizhanovskaya VV, et al., editors. Computational Science - ICCS 2020 Springer International Publishing; 2020. p. 481-495. 